E-Review Dossier 6-2018

Bologna (BraDypUS)

I molti territori della Repubblica fascista. Amministrazione e società nella RSI a cura di Roberto Parisini, Roberta Mira e Toni

Rovatti

\section{«Si spara sui fascisti e non sui prefetti!» \\ Tra ricerca del consenso e guerra contro la comunità: la silenziosa lotta tra lo Stato e il partito a Torino}

ISSN: 2282-4979

DOI: $10.12977 /$ ere-

view253

Il contributo analizza la Repubblica sociale italiana (Rsi) nel Torinese attraverso due aspetti: l'origine e l'evoluzione del conflitto interno tra Stato e partito e i rapporti esterni con la società del momento: comunità cittadina, industriali, Chiesa e tedeschi. La frattura tra agire politico e agire ideologico che emerge dentro il fascismo subalpino è la possibile chiave di lettura nell'esame dei rapporti tra la periferia e il centro della Rsi.

This essay analyzes the Italian Social Republic (Rsi) in the Turin area through two aspects: the origin and evolution of the internal conflict between state and party and the external relations with the contemporary society of the moment: the local community, the industry, the Church and the Germans. The gap between political action and ideological action that emerges within subalpine fascism is the possible key to understanding the relationship between the periphery and the center of the Rsi.

\title{
Introduzione.
}

\section{All'origine della diarchia: dall'alto verso il basso, dal centro alla periferia}

La figura del prefetto, con l'ulteriore articolazione del sottoprefetto, costituisce indubbiamente una collaudata cinghia di trasmissione tra il centro e la periferia (e viceversa) per tutto il periodo dello Stato liberale monarchico.

Con l'avvento del regime fascista e del partito unico, l'architettura preesistente inizia però ad essere modificata in senso autoritario. Per prima cosa, Mussolini 
attribuisce al prefetto il potere di nomina delle consulte municipali e dei podestà (leggi del 4 febbraio e del 3 settembre 1926) abolendo così i consigli comunali e la figura del sindaco. Si ripete dunque anche in periferia ciò che è già avvenuto a livello centrale, vale a dire la rottura del sistema "elezionista". [Lupo 2000, 216] Nello stesso tempo, sul versante del partito, l' 8 ottobre 1926 viene approvato il nuovo Statuto del Partito nazionale fascista (Pnf), che prevede un assetto basato sulle federazioni provinciali con a capo il segretario provinciale (poi divenuto segretario federale). Ad esso vengono subordinati i segretari dei singoli fasci di combattimento presenti in ogni comune della provincia. A sua volta, il segretario provinciale risponde direttamente al segretario generale del Pnf da cui è scelto'. Cessa così l'usanza abbastanza diffusa di nominare il segretario politico del fascio durante l'assemblea degli iscritti, spesso per acclamazione, e si impone anche qui un rigido sistema gerarchico che procede «dall'alto verso il basso» [Lupo 2000, 22]. Intanto, sul piano amministrativo, si registra in parallelo l'abolizione dei circondari (ente intermedio tra la provincia e il mandamento) con le relative sottoprefetture che li governano (Rdl 2 gennaio 1927, art. 3), trasferendo tutte le competenze alla prefettura. Il duce, nonché ministro dell'Interno ad interim, in una circolare del 5 gennaio 1927 ribadisce che il prefetto è «rappresentante diretto del potere esecutivo e centrale» ${ }^{2}$ nonché unico responsabile politico della provincia.

Queste trasformazioni creano, però, problemi crescenti man mano che il fascismo occupa segmenti importanti degli apparati statali, avviandosi a diventare un regime totalitario. La concorrenzialità tra Stato e partito innescata dalla diarchia prefetto/federale genera confusione e rivalità, tanto al centro quanto nelle periferie. Nella prima metà degli anni Trenta, le province diventano spesso terra di una prolungata e spesso silenziosa guerriglia tra prefetti e federali [Lupo 2000]. Per il momento, però, il ferreo controllo esercitato dal regime risulta in grado di assorbire le tensioni e governarle anche se i rapporti di forza sembrano lentamente volgere a favore del partito [Gentile 2001].

Una svolta importante si ha infatti con il Rdl 27 giugno 1937, n. 1058, che elimina il monopolio statale nella determinazione della carriera prefettizia, riducendo a $3 / 5$ il numero di prefetti immessi nella carica e tratti dalle carriere del ministero dell'Interno. La restante quota viene quindi destinata a chi proviene dalle fila del partito, spesso dalla carica di segretario federale o di ispettore di zona [Carucci 1993].

Mussolini alla Milizia e al Re nell'assumere il comando delle Camicie Nere, "La Stampa", 10 ottobre 1926.

Mussolini ai Prefetti: categoriche direttive politiche, "La Stampa", 6 gennaio 1927. 
Il quadro risulta profondamente mutato dopo 1'8 settembre 1943, con la nascita - sotto l'ingombrante tutela tedesca - dello Stato nazionale repubblicano, poi Repubblica sociale italiana (Rsi). Sparpagliato nelle sue sedi istituzionali in tutta l'area del Garda e anche oltre, assume ben presto una sorta di forma policentrica, in cui il duce è oramai solo uno degli attori, tutt'al più un primus inter pares. È in tale contesto che ritorna in discussione l'annosa questione della diarchia creata come si è visto - a metà degli anni Venti e mai risolta veramente.

Nel primo Consiglio dei ministri, tenuto il 28 settembre 1943, Mussolini ribadisce che solo «concentrando autorità e responsabilità in una sola persona, [si] ridarà al complesso delle nostre istituzioni locali la possibilità di un funzionamento per quanto possibile regolare» [Scardaccione (ed.) 2002]. Da questa direttiva, tradotta in riforma, nasce la figura del capo della provincia (giuridicamente la nomina è di prefetto e la qualifica di capo della provincia). In sostanza nulla di nuovo, tranne un aspetto: il reclutamento avviene esclusivamente all'interno del nuovo Partito fascista repubblicano (Pfr) in accordo tra il ministro dell'Interno e il segretario del partito [Rovatti 2011].

La concentrazione del comando politico e amministrativo nelle mani del capo della provincia, il quale - per la durata della guerra - sarà a capo tanto della Prefettura quanto della Federazione repubblicana fascista, è una riforma o innovazione che nel nostro sistema politico-amministrativo deve essere salutata con aperto compiacimento. [...] La riforma odierna [...] affida al capo della provincia il comando anche nel settore più propriamente politico: la organizzazione del Partito 3 .

La costante disgregazione politica e l'enormità dei problemi posti alla Rsi dalla guerra, su cui pesa sempre - occorre ricordarlo - l'occupazione tedesca, sembrano via via ridimensionare sia il ruolo del commissario federale (non più segretario federale), sia quello del capo della provincia. Quest'ultimo non è più in grado di far fronte alle complesse questioni derivanti dalla guerra, perché l'entità dei problemi va ben oltre i poteri e il territorio di competenza, basti pensare all'organizzazione del sistema dei trasporti o all'approvvigionamento alimentare. A ciò occorre aggiungere un altro aspetto: la militarizzazione del partito con la conseguente nascita delle Brigate nere sottrae al capo della provincia una parte importante della propria autorità. Infatti, pur essendone formalmente il comandante, sono i tedeschi a disporne.

Da questa enorme perdita di potere reale sul territorio sembra trarre origine - a partire dal settembre 1944 - la progressiva istituzione dei Commissariati straordinari, coincidenti con i singoli territori regionali ancora sotto il controllo della Rsi. 
Al vertice di ognuno viene posto un alto commissario che risponde direttamente a Mussolini e ha il potere di emanare atti aventi valore di legge, oltre a compiti di coordinamento sui capi delle province. Salta dunque il livello intermedio rappresentato dal ministro dell'Interno.

Qualcosa del genere va facendo anche il partito, trasformando via via la figura del delegato regionale in una sorta di super commissario federale che risponde direttamente al ministro-segretario del Pfr, Pavolini, e ha sotto di sé tutti i commissari federali della regione. La nuova figura appare dunque speculare all'alto commissario espresso dallo Stato.

In sostanza, dal piano provinciale la diarchia si proietta anche sul piano regionale.

\section{I tedeschi davanti a due opzioni: quella politica o quella fascista}

Dopo la sanguinosa occupazione di Torino e l'arresto del prefetto badogliano Vincenzo Ciotola, i tedeschi si mostrano attenti a non creare ulteriori tensioni nella comunità torinese che temono per la grande concentrazione operaia. Chiamano perciò alla guida della prefettura l'ingegner Angelo Tollini, un uomo che conosce la lingua tedesca, è legato alla Fiat ed è amico del console del Reich a Torino, il barone Dirk von Langen. Per gli occupanti questa è una scelta squisitamente politica, perché escludono volutamente i fascisti, odiati e screditati agli occhi della comunità, come si è visto il 26 luglio, puntando su un uomo non compromesso con il precedente regime, che anzi detesta i fascisti e motiverà la sua scelta di collaborare solo con il desiderio di mitigare i tragici effetti della presenza tedesca in città. Per certi versi, la sua figura e il suo atteggiamento politico costituiscono un osservatorio interessante in quanto ci mostrano la "normalità" di un paese occupato, cioè come sarebbe stata l'Italia senza una Resistenza e senza il ritorno sulla scena del fascismo repubblicano. Tollini rappresenta agli occhi dell'occupante una figura apolitica e moderata che dovrebbe fungere da mediatore con la comunità cittadina; il suo collaborazionismo è utile ai tedeschi per dispiegare in tutta tranquillità la propria politica di occupazione e di sistematica spoliazione. In effetti, essi lo reputano la scelta migliore, al punto da opporre una prolungata resistenza quando, in ottemperanza agli accordi presi ai livelli superiori, sono costretti a sostituirlo con un capo della provincia "politico" già nominato dal duce alla fine di settembre.

Dopo lunghe settimane di stallo, il 21 ottobre 1943 si insedia finalmente nel capoluogo piemontese (ultima fra le città della Rsi) il trentottenne Paolo Zerbino, già prefetto della provincia dalmata di Spalato. Il giorno dopo, la Militärkom- 
mandantur di Torino nella prima relazione mensile (Lagebericht) inviata alla sede centrale di Milano ribadisce che

l'atteggiamento della popolazione della provincia e della città è di sostanziale rifiuto con poche eccezioni, nei confronti del governo fascista [...]. A Torino, - ammonisce il documento - appoggiare apertamente i fascisti non gioverebbe affatto alla salvaguardia degli interessi tedeschi [Mantelli 1995, 160].

\section{La partita con gli industriali}

La riorganizzazione della presenza fascista in città inizia a porre un crescente problema anche per gli industriali, tra i quali è ancora vivo il ricordo delle grandi manifestazioni di piazza seguite alla caduta di Mussolini, che hanno visto protagonista la comunità torinese e in modo particolare la sua numerosa componente operaia. Quelle reazioni, ancora a distanza di mesi, sembrano esercitare involontariamente un certo condizionamento sugli industriali, suggerendo un atteggiamento di prudenza nelle relazioni in fabbrica.

Il nuovo regime, intanto, pur all'interno del ristretto spazio concesso dai tedeschi, appare ben deciso a giocare un ruolo e a riprendersi almeno una parte del potere perduto. Vi è però una significativa differenza con il passato: il fascismo repubblicano accusa ora apertamente la «plutocrazia» di aver sabotato la guerra e di aver impedito con ogni mezzo che il precedente regime attuasse politiche favorevoli alla classe operaia. La nuova situazione, secondo la lettura che ne danno i fascisti repubblicani, permetterebbe finalmente di realizzare quei programmi «politicamente meravigliosi» $»^{4}$, avendo come interlocutori non più gli industriali, visti come traditori, ma gli operai. Su queste basi propagandistiche, al principio dell'autunno 1943 prende forma una strategia di ricerca del consenso - di cui si occupa il partito - che probabilmente rispecchia anche quella di altre realtà industriali simili a Torino. Essa consiste, da un lato, nello sfruttamento della tradizionale conflittualità esistente tra operai e industriali e, dall'altra, nella legittimazione del fascismo repubblicano attraverso la crescita del suo potere contrattuale nei confronti degli altri soggetti della società torinese del momento, tedeschi compresi. Ciò sarebbe possibile grazie alla credibilità acquisita con la scelta anticapitalista e operaia.

È proprio in questa dimensione di affannosa ricerca del consenso che si inserisce - nella prima metà dell'ottobre 1943 - il grottesco tentativo del neocommissario federale, Giuseppe Solaro, di far arrestare dalle SS i vertici della Fiat, ossia il se- 
natore Giovanni Agnelli e l'amministratore delegato Vittorio Valletta. A suo dire, essi sarebbero «rappresentanti del capitalismo internazionale» e colpevoli di aver favorito «la nascita di consigli di fabbrica comunisti nel periodo badogliano». Come scrive il giovane dirigente del Pfr torinese, «con tali misure si potrebbe guadagnare quasi la metà delle maestranze» ${ }^{5}$.

L'iniziativa è però destinata ad un misero fallimento per la totale contrarietà dei tedeschi che non intendono affatto privarsi di due elementi di primo piano dell'industria italiana, considerati fondamentali per la produzione. Questo insuccesso, tuttavia, non interrompe minimamente l'offensiva propagandistica contro gli industriali torinesi che si dispiega anche con pressioni sul versante della stampa. Non può non colpire, ad esempio, in questo clima, l'ambiguo titolo apparso sul secondo numero del settimanale del partito di Torino, "La Riscossa", che in prima pagina, a caratteri cubitali, annuncia il «Socialismo!», mutando il precedente sottotitolo del giornale da "settimanale politico del popolo torinese" nel più classista "settimanale politico del popolo lavoratore". Il sottinteso per gli industriali è chiaro.

Intanto, un aiuto ideologico al nuovo corso "rivoluzionario" che va prendendo forma arriva in quelle settimane anche dal congresso di Verona (14-15 novembre 1943), che enuncia alcuni dei princìpi che dovrebbero realizzare la socializzazione delle imprese, definita dai sindacalisti fascisti la terza via tra il capitalismo e il comunismo.

La propaganda da un lato e il nuovo corso ideologico del Pfr dall'altro non sembrano però tener più conto della nuova realtà, del diverso quadro politico-militare e soprattutto dei mutati rapporti di forza rispetto ai tedeschi, al punto da far apparire anacronistica l'idea stessa del fascismo repubblicano di riproporsi in quel ruolo di mediazione tra operai e industriali avuto fino al luglio del 1943, sia pure abbracciando ora la causa dei lavoratori. Un banco di prova delle trasformazioni avvenute con il 25 luglio è costituito proprio dai massicci scioperi spontanei contro la fame e per l'aumento delle paghe, che investono Torino tra il 22 novembre 1943 e gli inizi di dicembre. In quella circostanza, i fascisti sperimentano la chiusura degli spazi e tutta l'ostilità accumulata nelle fabbriche, in cui spesso i

Archivio di Stato di Torino (d'ora in poi ASTo), Sezione Corte, fondo Sandretti, b. 21, f. 2, memoriale di Vittorio Valletta, 15 agosto 1945, lettera inviata al Comando Superiore per I'Italia dal maggiore delle SS Herbert Kappler, 10 ottobre 1943. II documento, in copia fotografica, figura allegato al memoriale.

6 "La Riscossa", 4 novembre 1943.

(fr. ASTo, Sezioni riunite (d'ora in poi SR), Gabinetto di Prefettura (d'ora in poi GdP), b. 550, Specchietto delle ore lavorative perdute fino al 3 marzo, s.d. Dal promemoria risulta che a Torino nei giorni successivi al 22 novembre 1943 sono 71.395 gli operai in sciopero per un numero complessivo di giornate lavorative perdute pari a 61.891; a dicembre, i lavoratori in sciopero sono 14.300 per un numero complessivo di giornate perdute pari a 7.612,5. 
delegati sindacali del regime non riescono neppure ad entrare. Sono invece i tedeschi a giocare un ruolo e a porsi come interlocutori riconosciuti e temuti dagli operai. Ma non sono solo l'ostilità e il discredito a mettere nell'angolo il partito: occorre infatti aggiungere un altro aspetto su cui torneremo più avanti, vale a dire l'avvio di quel lungo scontro con lo Stato - rappresentato dal capo della provincia Zerbino - iniziato in quelle stesse settimane, proprio sul terreno della ricerca del consenso all'interno della più ampia comunità cittadina e della strategia politica da adottare per conseguirlo.

Questa situazione, unitamente ai precedenti con cui il federale Solaro ha fatto la sua apparizione sulla scena cittadina, ossia cercando di far arrestare i vertici della Fiat, non fa che confermare all'interno del mondo degli industriali che non vi è più alcuna convenienza nel collaborare con il fascismo, ormai condannato alla sconfitta. Si apre perciò una partita pericolosa e complicata in cui l'orientamento prevalente che si fa strada tra gli imprenditori sembra essere per ora duplice. Da un lato, quello di evitare di tenere rapporti troppo stretti con gli occupanti tedeschi per non compromettersi eccessivamente agli occhi della comunità cittadina e della sua componente operaia in particolare; dall'altro, cercare di mantenere un atteggiamento di apparente collaborazione con le autorità della Rsi, ossia Stato e partito, che insistono per recuperare, ad esempio, il vecchio ruolo di rappresentanza degli operai o di mediazione nel controllo dei flussi della manodopera, oltre a spazi per la propaganda e contributi economici per le attività del nuovo regime. Possiamo immaginare che il colloquio del 18 dicembre 1943, tra Mussolini e Valletta, convocato per fornire spiegazioni in merito agli scioperi appena conclusi, costituisca proprio uno dei momenti di avvio di questa lunga strategia della dissimulazione con cui gli industriali cercano di limitare i danni, dilazionando le pressanti richieste fasciste con pretesti più o meno credibili, aventi lo scopo di guadagnare tempo e arrivare alla fine della guerra ${ }^{8}$.

I fascisti repubblicani, dal canto loro, appaiono consapevoli della politica assunta nei propri confronti dal mondo dell'industria. Proprio in quei giorni, in un suo rapporto al ministro Pavolini, il commissario federale Solaro scrive senza mezzi termini che occorre «non avere dei particolari riguardi verso gli industriali torinesi $[\ldots]$ il cui comportamento è assolutamente equivoco»?. 


\section{L'agire politico dello Stato contro l'agire ideologico del partito}

Le norme sull'unità di comando ispirate dal duce, non sembrano sufficienti ad appianare i contrasti che lacerano molte periferie della Rsi. A Torino, intanto, come si è detto, mentre si sviluppano gli scioperi dell'autunno 1943, si consuma un lungo scontro tra il capo della provincia e il commissario federale Solaro. Sia pure in un quadro assai diverso, si ripropone nuovamente quella "guerriglia" fra le due cariche già vista negli anni Trenta, ma che ora il centro non riesce più ad assorbire. Se le linee da seguire nella ricerca del consenso risultano chiare nella schematicità con cui le ha tracciate Mussolini con il discorso da Radio Monaco del 15 settembre 1943 e sintetizzabili nella formula «assistere, lavorare, combattere», meno chiaro è quale sia la strategia da applicare per il raggiungimento degli obiettivi e chi debba imporla. Si tratta di un'ambiguità di non poco conto che riflette l'estrema debolezza della compagine della Rsi. Priva di grandi mezzi e apparati solidi, fortemente compressa dall'alleato-occupante, essa delega implicitamente ogni decisione alle variegate realtà periferiche e ai rapporti di forza ivi presenti. Nella grande incertezza del momento è però il partito a mettere a segno un importante colpo a livello nazionale su un aspetto che più di ogni altro dovrebbe creare consenso: in sostanza, approfittando delle difficoltà in cui versa lo Stato in quel momento, riesce ad ottenere dal ministro dell'Interno, Buffarini-Guidi, il monopolio dell'intero settore dell'assistenza attraverso l'eliminazione degli Enti comunali di assistenza e la creazione degli Enti provinciali di assistenza fascista (12 novembre 1943) [Adduci 2014, 94-95], cosa che non era riuscita nemmeno al Pnf. Si tratta di una forte arma di pressione che inizia ad essere usata come merce di scambio per la creazione di un consenso forzato un po' dappertutto. Si pensi a quanto avviene nel Torinese, dove ad esempio gli aiuti ai bisognosi vengono erogati solo se in paese è stato aperto un fascio repubblicano e solo se la popolazione si mostra favorevole al regime ${ }^{10}$.

A Torino, almeno inizialmente, Stato e partito avviano attività assistenziali congiunte, ma si tratta di aiuti estemporanei e insufficienti che paradossalmente finiscono con l'alimentare la già fortissima avversione nei confronti della guerra", dei tedeschi e della stessa Rsi. Al suo interno, la componente dello Stato mostra

Cfr. ASTo, SR, GdP, b. 33/2, f. Sospensioni servizio assistenza, lettera di Giuseppe Solaro al commissario prefettizio di Orbassano, 3 febbraio 1944.

Nella comunità cittadina essa si traduce in un'alta renitenza alla leva, basti pensare che nel solo Distretto militare di Torino, tra i giovani della classe 1925, si arriva al 57,2\% di assenti ingiustificati alla chiamata. Cfr. ASTo, SR, GdP, b. 145, comunicazione al capo della provincia dei dati sul reclutamento della classe 1925 nel Distretto militare di Torino, 15 febbraio 1944. 
sempre più diffidenza verso il partito che - in nome della "purezza" rivoluzionaria di cui sarebbe portatore - pratica una violenza crescente contro la comunità torinese, che non solo annulla gli sforzi un po' velleitari messi in atto dalla componente statale per ottenere il consenso, ma genera problemi di ordine pubblico anche gravi [Adduci 2014, 84].

In un simile quadro, fatto di contrapposizioni e dissidi, se si esclude la determinazione nel proseguire la guerra al fianco dei tedeschi, sono davvero pochissimi gli altri aspetti su cui Stato e partito sembrano convergere pienamente: la spietata persecuzione degli ebrei ${ }^{12} \mathrm{e}$ l'avvio della socializzazione sono però certamente fra questi.

Per il resto, il Pfr del federale Solaro, in sintonia con Pavolini - e contrariamente a ciò che aveva fatto il Pnf - si caratterizza per un agire ideologico che lo porta ad abbandonare ben presto ogni ricerca del consenso, in una crescente condizione di "estraneità" dalla comunità. Tra i dirigenti del partito riprende forza l'idea già circolante negli ultimi anni del precedente regime, ossia la necessità di una fascistizzazione dello Stato, giudicato non all'altezza della situazione [Gentile 2001]. L'operato di Zerbino, che trova momentaneamente una sponda anche nei tedeschi di stanza in città, sempre preoccupati della forza numerica degli operai, si caratterizza invece per un agire politico, fatto di pressioni (per esempio sulla Chiesa subalpina affinché intervenga con la propria autorità per modificare la moralità dei giovani renitenti), sanzioni mirate contro i familiari dei renitenti, propaganda, trattative ma anche dure quanto astute forme di repressione del nascente movimento partigiano. Egli ricorre al doppio gioco e all'azione disgregatrice, incoraggiando gli scontri armati tra le prime bande partigiane formate da ex militari e quelle comuniste, così come ha visto fare in Jugoslavia durante le operazioni di intelligence e controguerriglia del Regio esercito. Sistemi che gli consentono, ad esempio, nel dicembre 1943, di smantellare senza colpo ferire il primo Comitato militare regionale piemontese (Cmrp) che dirige la Resistenza.

Questo agire viene però interpretato dal partito come debolezza. La visione politica di Solaro, sia nei confronti dei renitenti, sia nei confronti della nascente Resistenza di qualunque colore, passa attraverso la criminalizzazione dell'intera

Con I'Ordine di polizia n. 5, del 30 novembre 1943, il ministro dell'Interno Buffarini-Guidi ordina ai capi delle province di arrestare immediatamente tutti gli ebrei, disponendone l'invio in campi di concentramento provinciali. A Torino, una delle prime azioni di fermo ad opera della polizia si registra con l'arresto di 20 anziane donne ebree ricoverate nell'ospizio comunale di via Como 140 (ASTo, SR, fondo Casa circondariale Le Nuove, Registro matricola, 1943). II partito, invece, si concentra sulla propaganda: quasi in contemporanea, il settimanale "La Riscossa", dà la notizia dei prowedimenti presi dalla Rsi, accompagnandola con un'odiosa vignetta razzista pubblicata in grande evidenza sulla prima pagina (Gli ebrei finalmente serviti!, "La Riscossa", 2 dicembre 1943). 
comunità, considerata sempre di più collusa e dunque meritevole di rappresaglie indiscriminate, cosa che il capo della provincia, per ragioni esclusivamente politiche, vorrebbe invece circoscrivere il più possibile.

Sono dunque contrapposti un agire politico paternalista, spregiudicato e pericoloso per la Resistenza e un agire ideologico, di cui si è già detto, che allarga a dismisura il fronte dei nemici e trascina rapidamente il partito da una condizione di estraneità dalla comunità, ad una di alterità. Queste tensioni, esplose tra il gennaio e il febbraio 1944, portano solo ad un momentaneo «chiarimento provocato dalla Direzione del Partito, che chiamò al Quartier Generale, Prefetto e Federale» $»^{13}$.

Nel campo fascista - scrive al duce un alto funzionario del Pfr - il Prefetto è molto
mal visto e accuse di ogni genere vengono mosse contro di lui. [...] L'attività svol-
ta [...] sarebbe una politica di accomodamento e di compromessi, che non rientre-
rebbe assolutamente nel clima rivoluzionario del momento, ed infatti non è raro il
vedere elementi conosciuti come ostili o per lo meno poco favorevoli al Fascismo,
intrattenersi a lungo nelle sue anticamere ed anche con lui. [...].
Fin dai primi giorni [...] si delineò una netta divergenza sul terreno dell'azione
politica da condurre fra Prefetto e Federale. Il Federale intendeva svolgere una
energica azione di epurazione in città ed in provincia e chiese l'immediata sosti-
tuzione del Questore [...]. Il Prefetto intendeva viceversa condurre una politica di
avvicinamento, che è considerata titubanza e mancanza di energia dall'elemento
fascista ${ }^{14}$.

Il policentrismo della Rsi si riflette bene nelle enormi difficoltà incontrate dal capo della provincia, non solo a Torino, nell'affermare la piena autorità nei confronti del partito che appare sempre più fuori controllo e con i suoi sistemi minaccia di trascinare la componente statale verso la guerra civile.

Al ministro dell'Interno e allo stesso duce, i capi delle province chiedono a più riprese un deciso intervento per far rispettare i princìpi gerarchici «dall'alto verso il basso [...] dal centro verso la periferia» [Lupo 2000, 22], a cui li ha formati in passato il fascismo. Ma il centro, ossia la compagine della Repubblica sociale, è fragile e non risulta in grado di assorbire come faceva prima le tensioni provenienti dalle periferie. Ne dà una conferma indiretta lo stesso Mussolini, che in un duro telegramma inviato nel febbraio 1944 a tutti i capi delle province, circa i problemi di ordine pubblico causati dalle squadre armate del Pfr, rimanda senza mezzi termini la soluzione alle dimensioni locali: «Bisogna che i Capi delle Pro- 
vincie (sic) si ricordino che sono anche i Capi del Partito e invece di rivolgersi continuamente al Ministero [...] risolvano in loco le situazioni $[\ldots] »^{15}$.

Nel pieno di questo conflitto interno e delle tensioni crescenti con la comunità, il 12 febbraio 1944 giunge intanto l'approvazione del decreto legislativo sulla socializzazione $^{16}$, uno dei pochissimi aspetti su cui - come si è detto - Stato e partito sembrano trovare una piena convergenza. Anche se mancano ancora i decreti applicativi, il provvedimento viene speso ampiamente dalla propaganda come il passo decisivo a favore della classe operaia e contro gli industriali e il capitalismo. E questa volta sono i tedeschi, assai preoccupati, a convocare Valletta per un chiarimento con Rahn, ambasciatore e plenipotenziario del Reich presso la Repubblica sociale ${ }^{17}$.

\section{4. «Si spara sui fascisti e non sui prefetti!»: la ricerca della terzietà come strategia per sottrarsi alla guerra contro la comunità}

Le opposte strategie sembrano trovare un altro momento di sia pur parziale convergenza in occasione degli scioperi politici del marzo 1944, quando le forze di polizia della Rsi arrestano circa 400 lavoratori prontamente consegnati ai tedeschi per l'invio in Germania. Ma anche in questa circostanza, dal partito e dalla Guardia nazionale repubblicana entrata nella sua orbita, non mancano le voci critiche e le accuse nei confronti di Zerbino e della dipendente Questura, accusati di aver sottovalutato quanto stava accadendo e di essersi mossi troppo tardi nella repressione [Comitato Torinese per le Celebrazioni del 50ennale della Liberazione (ed.) 1994, 54-61] ${ }^{18}$.

È l'ultimo campanello d'allarme prima del punto di non ritorno raggiunto poche settimane dopo, in coincidenza con due avvenimenti: l'uccisione del fascista repubblicano Ather Capelli, condirettore della "Gazzetta del Popolo", ad opera dei Gruppi d'azione patriottica (Gap) e il processo contro i dirigenti del movimento di resistenza piemontese, cioè il generale Perotti e gli altri componenti del se-

15 ASTo, SR, GdP, b. 33/1, f. Polizia interna federale, trascrizione del telegramma n. 954 del 15 febbraio, diretto dal duce a tutti i capi delle province, 26 febbraio 1944.

16 La socializzazione della struttura economica del Paese, "La Stampa", 13 febbraio 1944.

17 ACS, SPD, CR, RSI, 1943-1945, b. 27, Promemoria del capo della provincia per il duce, 15 febbraio 1944. La visita awiene il 10 febbraio.

18 Relazione del console capo della I Legione Gnr, Gaetano Spallone al Comando generale, 28 marzo 1944, documento riprodotto. 
condo Cmrp, arrestati il 31 marzo 1944 in duomo, poco prima di una riunione clandestina.

Mentre il partito, ancora scosso dall'azione contro Capelli [Adduci 2014, 198]', chiede rabbiosamente la pena di morte per tutti i quindici arrestati, dopo un processo istruito e chiuso a tempo di record, in Camera di consiglio sembra farsi strada ancora una volta l'agire politico.

Gli otto condannati a morte sono due alti ufficiali, considerati pericolosi per le loro elevate competenze tecniche nonché per il ruolo rivestito nel movimento di resistenza e nel Cmrp, e sei militanti o simpatizzanti dei partiti di sinistra. Vengono risparmiati, invece, due ufficiali superiori giudicati marginali nonché gli esponenti cattolici e liberali, oltre ad un vecchio socialista che si pensa (inutilmente) di "utilizzare".

È in sostanza questo il momento in cui lo Stato - pur in una dimensione fortemente autoritaria - tenta di definirsi pubblicamente sulla base di un sempre più marcato ruolo di terzietà, da giocare tra le parti in lotta preservando il monopolio della violenza [Ganapini 1999, 275]. Il partito, invece, attraverso la Gnr prima e le Brigate nere poi, punta decisamente alla guerra totale contro la comunità, identificata ormai in toto con la Resistenza, come dimostrano i sanguinosi rastrellamenti in Val Pellice, la rappresaglia di San Maurizio Canavese e le azioni condotte con i tedeschi nei mesi precedenti. A questi episodi, la Resistenza risponde via via incrementando le uccisioni di commissari del fascio e di militi isolati. Ma non mancano neppure i podestà e i commissari prefettizi. Negli ambienti del partito, però, al centro come nelle periferie della Rsi, l'isolamento e la perdita del senso della realtà iniziano ad apparire evidenti anche nell'errata percezione che la propria organizzazione sia l'unico bersaglio delle forze partigiane, mentre gli apparati statali godrebbero di una sorta di immunità.

«Si spara sui fascisti e non sui prefetti! $»^{20}$ - che dunque non sarebbero fascisti - si sente ripetere sempre più spesso tra i militanti del Pfr.

La terzietà, che non significa certo neutralità, fa riferimento probabilmente ad un sentire diffuso piuttosto presente negli apparati torinesi dello Stato e si delinea per molti mesi come una vera e propria strategia per evitare di essere risucchiati nella guerra contro la comunità.

Senza alcun processo, la mattina del 2 aprile 1944, cinque partigiani - tratti dalle Nuove - vengono fucilati nel cortile dell'abitazione del giornalista e lasciati esposti in strada per alcune ore.

20 ACS, SPD, CR, RSI, 1943-1945, b. 11, f. Torino, relazione con oggetto: situazione politica di Torino e del Piemonte, s.d., foglio 046707 
Su questa base sembra realizzarsi una continuità anche quando il 12 maggio 1944 Zerbino è sostituito da Edoardo Salerno, già capo della provincia di Roma, sprezzantemente definito dai fascisti torinesi un «legalitario» [Rovatti 2011].

Se da un lato il suo atteggiamento risulta debole perché non si scontra mai direttamente con Solaro, dall'altro egli conforma quasi sempre la propria azione al rispetto assoluto e formale delle norme, sinonimo di ordine da contrapporre al disordine e al caos generato dal partito (e dai tedeschi), ormai percepiti dalla comunità come la stessa cosa, ossia stranieri.

La sua attenzione nel mantenersi in questa posizione di terzietà, emerge in forma clamorosa il 25 luglio 1944, in occasione della prima adunata pubblica della Brigata nera torinese che celebra i propri riti costitutivi in una caserma del centro. Al termine della cerimonia, così come sono soliti fare e ottenere, $i$ fascisti chiedono a gran voce di sfilare in corteo per le vie di Torino, in una sorta di simbolica riconquista di quello spazio pubblico ormai precluso, ma Salerno (che pure ha partecipato alla manifestazione in quanto capo della provincia) oppone un deciso rifiuto per non alimentare nuove tensioni con la comunità.

«Chiedemmo di poter sfilare per la città - scrive l'allora giovane ausiliaria Marazio - ma non ci fu concesso per evitare possibili disordini» [Marazio 1998, 162].

\section{Pane e lavoro}

Oltre a questo aspetto, che - si è detto prima - appare assolutamente privo di significato agli occhi della comunità cittadina, Salerno deve dedicare sforzi enormi per garantire un minimo di rifornimenti alimentari per Torino. Nell'estate del 1944, la favorevole congiuntura data dalla stagione, permette per un paio di mesi un certo afflusso di viveri in città, anche se - occorre ricordarlo - si tratta di quantità assolutamente insufficienti. Non si può ignorare che la questione alimentare sia fondamentale non solo per il consenso ma anche per la tenuta dell'ordine pubblico. La fame, come istanza non rimandabile proveniente dalle periferie, e Torino ne soffre particolarmente, sollecita sempre più una decisione dagli organi centrali della Rsi che finora sono apparsi incapaci di affrontare la questione in modo efficace.

Dal canto loro, sia la Chiesa, sia gli industriali giocano una partita analoga. Questi ultimi, in particolare nelle dimensioni medio-grandi, così come avveniva in passato, imboccano la strada di un'autonoma ricerca del consenso tra i lavoratori dei propri stabilimenti, attraverso concessioni crescenti come gli spacci aziendali a prezzi calmierati, alcune distribuzioni gratuite di beni introvabili sul mercato, si pensi alle gomme per le biciclette, gli anticipi in busta paga e via dicendo. Si trat- 
ta di vere e proprie provvidenze che divengono una sorta di reddito integrativo; dietro il paravento della collaborazione con le autorità fasciste «per alleviare le condizioni delle masse operaie» si nasconde in realtà una concorrenza con la Rsi, che esce ampiamente screditata visto che non riesce a garantire neppure i generi previsti dalla tessera.

Non potendo tenere il passo con le risorse messe in campo in molti grandi stabilimenti, il governo fascista, già a metà del giugno 1944, gioca una carta propagandistica a costo zero, obbliga cioè le aziende industriali con meno di 500 lavoratori a costituire la mensa aziendale per i propri dipendenti e ad aprire spacci aziendali o interaziendali entro due settimane ${ }^{21}$. Si raggiunge così un duplice obiettivo: in primo luogo si aumenta l'accesso della classe operaia a risorse alimentari e beni primari che lo Stato non può garantire, scaricando i costi sulla piccola e media industria; in secondo luogo il merito dell'iniziativa (e il relativo consenso) sono tutti della Rsi.

A Torino Salerno emana subito il decreto di applicazione del provvedimento e contemporaneamente, in accordo con la Sezione provinciale per l'alimentazione (Sepral) prova a tamponare per lo meno le situazioni tragiche che più di altre "fanno opinione" nel desolato panorama alimentare di quel momento. In questo quadro, spende le poche risorse per attuare un provvedimento rivolto ad un certo numero di famiglie numerose del Torinese, a cui garantisce un piatto di minestra «per un massimo di 5000 razioni giornaliere»" ${ }^{22}$ una goccia nell'oceano. La ricerca del consenso passa in buona parte anche attraverso il cibo, ne è un esempio l'istituzione delle "Minestre del popolo" servite nelle scuole - trasformate in mense durante la chiusura estiva - a un prezzo politico e associate alla figura di Salerno che spesso si reca in visita al momento dei pasti, quasi per ricordare con la sua presenza chi si deve ringraziare se quel giorno si mangia qualcosa.

Le forti pressioni esterne esercitate dai tedeschi e dai fascisti sembrano intanto realizzare un aspetto paradossale: una condizione conflittuale come quella esistente tra gli industriali e la classe operaia finisce per trasformarsi lentamente in una coincidenza di interessi che pone al centro la salvaguardia dei macchinari e, in seguito, la difesa degli stabilimenti e degli impianti per la produzione dell'energia elettrica. L'avvicinamento tra i due soggetti registra un'accelerazione nel giugno 1944, in seguito all'ordine impartito dai tedeschi di smontare e trasferire i macchinari dell'Officina 17 della Fiat Mirafiori nelle gallerie della Gardesana,

La corresponsione di 8 lire al giorno ai lavoratori privi di mensa aziendale, "La Stampa", 13 giugno 1944 
forse in previsione di uno sbarco alleato in Liguria che avrebbe potuto tagliar fuori il Piemonte, determinando così la perdita dell'importante produzione torinese di motori aeronautici. Il timore degli operai scesi in sciopero, oltre ad un trasferimento così lontano che forse nasconde anche altro, è quello che i macchinari possano finire definitivamente in Germania, preoccupazione condivisa dai vertici della Fiat. Ad un riluttante Valletta, i tedeschi ordinano di riunire i lavoratori non per la questione dei macchinari, ma per «calmare gli stessi e convincerli che qualora fossero stati trasferiti essi non sarebbero andati in Germania». Ma è il presidente della Fiat che sembra gettare un ponte con gli operai, come annotano le autorità di polizia: «Interveniva anche il Senatore Agnelli che, presa la parola e manifestato il suo disappunto per quanto andava accadendo, riscuoteva dalla massa presente il totale plauso e l'unanime consenso» ${ }^{23}$.

Mentre le autorità statali della Rsi tacciono imbarazzate e scoppiano altri scioperi spontanei che minacciano di allargarsi a macchia d'olio, gli operai tedeschi della Todt, il 21 giugno, iniziano a smontare i macchinari da trasferire [Luraghi 1958, 227-230; Castronovo 2003, 479-481] $]^{24}$. Come è già accaduto in concomitanza con altri momenti di tensione nelle fabbriche, il partito, che ha smesso da mesi di fare politica, in quel momento sembra preoccuparsi più che altro delle vicende che lo attraversano al proprio interno e della militarizzazione ormai in atto che di lì a breve porterà alla nascita delle Brigate nere. La questione degli scioperi, quindi, ha sì un suo spazio, ma limitato alla dimensione di un supporto alle richieste dei tedeschi e alle eventuali ripercussioni sul partito travagliato da una crisi interna tra le varie anime. È in questa situazione che il 23 giugno giunge a Torino, per una visita di tre giorni, il capo della segreteria politica del Pfr, Olo Nunzi, inviato da Pavolini per un'inchiesta sulla situazione in città, che ha però come perno proprio le tensioni scoppiate dentro il partito [Gagliani 1999, 93] $]^{25}$.

\footnotetext{
ACS, Ministero dell'Interno, Direzione generale pubblica sicurezza, Divisione affari generali e riservati, Segreteria del Capo della Polizia, RSI, 1943-1945, b. 63, relazione quindicinale del commissario capo di Ps del Settore di Torino all'Ispettorato generale di polizia speciale di Milano, 29 giugno 1944. 


\section{Lo Stato come il partito}

L'esplosione violenta della lotta partigiana, i bombardamenti, la difficoltà nelle comunicazioni e l'impossibilità di regolare su base provinciale problemi enormi come l'approvvigionamento alimentare della popolazione o la pianificazione dei trasporti, su cui la Rsi si gioca una notevole fetta di consenso, mettono in luce tutta l'inadeguatezza della figura dei capi della province all'interno della nuova, angusta dimensione venuta a crearsi. È ormai sotto gli occhi di tutti la quotidiana e drammatica concorrenza in atto fra loro per l'accaparramento di pochi rifornimenti alimentari che sovente sfocia in tensioni interprovinciali anche per il rifiuto (e talvolta l'impossibilità per mancanza di mezzi) di esportare le eccedenze, cosa che genera non solo divisioni tra le autorità, ma anche e soprattutto forti malumori nelle varie comunità, in particolar modo quelle cittadine, maggiormente esposte alla fame in quanto dipendenti dalle campagne. Pesa inoltre in maniera notevole e costante la sistematica rapina di risorse alimentari effettuata dai tedeschi con l'invio continuo di convogli verso il Brennero su cui nessuno può intervenire. Così come nessuno, neppure la compagine della Rsi, può fermare le tradotte cariche di uomini rastrellati nelle valli e in città che vengono mandati nei campi di lavoro del Reich.

In questa situazione drammatica, la saldatura di interessi tra industriali e operai per la salvaguardia delle risorse materiali, iniziata con la vicenda della Gardesana, sembra allargarsi anche ad un altro aspetto, vale a dire la preservazione delle risorse umane. Questa trasformazione farebbe pensare ad una collusione crescente che supera la semplice dimensione di fabbrica e si allarga ad ampi settori della comunità cittadina, in sostanza il soggetto che esprime pienamente la Resistenza. Un esempio di questa inedita collaborazione è dato dal fenomeno delle massicce assunzioni di giovani partigiani o anche semplici renitenti, che trovano nelle fabbriche un rifugio sicuro dai tedeschi e dai fascisti in coincidenza con il momento più difficile per la lotta di liberazione, vale a dire l'autunno-inverno 1944-45. Si tratta della salvaguardia di giovani risorse, utili per riprendere la lotta partigiana con la primavera e al tempo stesso garantire un futuro alla comunità dopo la guerra.

Intanto, per frenare il processo di disintegrazione degli organi periferici dello Stato, il duce accoglie le istanze di maggiore autonomia provenienti dalla periferia e istituisce - nel settembre 1944 - i Commissariati straordinari, a partire da quello per il Piemonte. È il tentativo con cui il centro, per permettere la sopravvivenza dello Stato, delega ampi poteri venendo meno ad uno dei cardini del fascismo, ossia l'accentramento e il principio dal centro alla periferia. 
A capo pone il sottosegretario all'Interno, Zerbino, che il 21 settembre fa così ritorno in città nella veste di alto commissario con il compito di sovrintendere $\mathrm{e}$ coordinare l'azione dei capi delle province. Di lì a breve, intanto, giunge alla guida della prefettura di Torino il quarantacinquenne Emilio Grazioli [Rivello 2002, $177]^{26}$, noto per essere stato già alto commissario della provincia di Lubiana, dove ha governato con il pugno di ferro.

L'istituzione di questo nuovo soggetto, pur essendo una scelta dettata da una situazione di emergenza, non costituisce un provvedimento isolato; al contrario, si inserisce in un progetto più ampio, mirante al recupero di una certa funzionalità dello Stato attraverso una semplificazione delle proprie strutture e un maggior coordinamento che dovrebbe restituire infine anche un minimo di prestigio, in primis verso la comunità. In realtà, tutta l'operazione appare non solo irta di ostacoli ma anche fortemente contraddittoria per il mantenimento della contrapposizione fra centralismo e autonomia, ossia tra indirizzi generali e specificità locali fortemente esasperate dalla guerra e di cui non si tiene conto [Ganapini 1999]. Il ritorno di Zerbino, che lascerebbe presumere la ripresa con più energia di una linea basata sull'agire politico, non sembra invece rispondere alle aspettative dei suoi: l'offensiva partigiana ha toccato l'apice e le violenze, i rastrellamenti e le stragi compiute dai tedeschi e dai fascisti hanno di fatto reso impossibile ogni rapporto con la comunità. A ciò occorre aggiungere il cambio quasi completo dei vertici militari del gruppo dirigente tedesco di Torino, che dimostra immediatamente un atteggiamento simile a quello di Solaro e delle Brigate nere. Il 12 ottobre 1944, in seguito ad un attentato partigiano, avvenuto in piazza Statuto contro un locale frequentato da tedeschi, viene subito eseguita in piena notte una rappresaglia con nove fucilazioni in strada [Adduci et al. 2015, 93-94].

A Torino gli stessi apparati statali, che fino a questo momento sono riusciti a conservare dietro la facciata legalitaria una posizione di terzietà rispetto alla lotta in corso, iniziano ad essere sempre più risucchiati verso il confronto militare totale imposto dal partito, da cui tentano - non senza contraddizioni - di separare invano le proprie sorti, sperando con ciò di sottrarsi alla resa dei conti e dare continuità allo Stato: a quello Stato, attraverso i suoi uomini e i suoi apparati anche dopo il crollo della Rsi.

Con la nomina di Zerbino a ministro dell'Interno, avvenuta nel febbraio 1945, la figura di Grazioli viene ad assumere un'importanza notevole perché ottiene anche 
la carica di alto commissario. La sua azione politica risulta schiacciata sulle posizioni del partito, come d'altronde quella di Zerbino sia nella veste di alto commissario che di ministro. Le continue serrate delle fabbriche, decretate per punire ogni minimo sciopero, sono in piena sintonia con l'atteggiamento del partito, che se da un lato sbandiera l'avvio della socializzazione grazie ai decreti attuativi, con cui dovrebbe conquistare il consenso della classe operaia; dall'altro, conduce con la Brigata nera sanguinosi rastrellamenti, alimentando una guerra civile che si combatte in larga parte proprio contro quella componente che vorrebbe avere dalla propria parte.

Ma il conflitto tra Stato e partito, che sembra in cima alle preoccupazioni della compagine della Rsi, non ha alcun significato per la comunità cittadina e per gran parte della società torinese, che ormai da molto tempo non guarda alle sottili distinzioni portate avanti con la terzietà.

Nonostante le sue profonde divisioni, la Rsi appare (ed è) una sola nella sua funzione di repressione e di collaborazione con i tedeschi, come risulterà chiaro alla resa dei conti.

Il presente contributo è una rielaborazione rivista ed ampliata della relazione presentata al convegno "I molti territori della Repubblica fascista. Amministrazione e società nella RSI", Ferrara 27/28 settembre 2017. A fronte dell'impossibilità di sottoporre il testo ad un processo di double blind peer-review, i curatori hanno optato per una revisione preliminare da parte del relativo discussant di sessione.

\section{Bibliografia}

Adduci N. et al. 2015, Che il silenzio non sia silenzio. Memoria civica dei caduti della Resistenza a Torino, Torino: Museo Diffuso della Resistenza e Istituto piemontese per la storia della Resistenza e della società contemporanea (I ed. 2003).

Adduci N. 2014, Gli altri. Fascismo repubblicano e comunità nel Torinese (1943-1945), Milano: FrancoAngeli.

Carucci P. 1993, Il Ministero dell'Interno: prefetti, questori, ispettori generali, in Ventura A. (ed.) 1996, Sulla crisi del regime fascista 1938-1943. La società italiana dal «consenso» alla Resistenza, Annali 1992-95 - Istituto Veneto per la storia della Resistenza, Padova: Marsilio.

Castronovo V. 2003, Giovanni Agnelli: il fondatore, Torino: Utet (I ed. 1971).

Gagliani D. 1999, Brigate nere. Mussolini e la militarizzazione del Partito fascista repubblicano, Torino: Bollati Boringhieri.

Ganapini L. 1999, La repubblica delle camicie nere, Milano: Garzanti. 
Gentile E. 2001, La via italiana al totalitarismo. Il partito e lo Stato nel regime fascista, Roma: Carocci [ed. org. 1995].

Comitato Torinese per le Celebrazioni del 50.le della Liberazione (eds.) 1994, Gli scioperi del marzo 1944, Torino.

Lupo S. 2000, Il fascismo: la politica in un regime totalitario, Roma: Donzelli.

Luraghi R. 1958, Il movimento operaio torinese durante la Resistenza, Torino: Einaudi.

Mantelli B. 1995, L'occhio del padrone. I rapporti mensili della Militärkommandantur a Torino, in Boccalatte L., De Luna G. e Maida B. (eds.) 1995, Torino in guerra, Torino: Gribaudi.

Marazio Z. 1998, Il mio fascismo. Storia di una donna, Baiso: Verdechiaro Edizioni.

Rivello P. 2002, Quale giustizia per le vittime dei crimini nazisti?, Torino: Giappichelli.

Rovatti T. 2011, Leoni vegetariani. La violenza fascista durante la RSI, Bologna: Clueb.

Scardaccione F.R. (ed.) 2002, Verbali del Consiglio dei ministri della Repubblica sociale italiana, Roma: Ministero per i Beni e le Attività culturali - Direzione generale per gli archivi. 